\title{
Investigating nystagmus in patients with traumatic brain injury: A systematic review (1996 - 2016)
}

\author{
H de Clercq, MCommunication Pathology (Audiology); A Naudé, PhD; J Bornman, PhD \\ Centre for Augmentative and Alternative Communication, Faculty of Humanities, University of Pretoria, South Africa
}

Corresponding author: $H$ de Clercq (hendrika@hdcinc.co.za)

\begin{abstract}
Background. Traumatic brain injury (TBI) is a health and socioeconomic concern worldwide. In patients with TBI, post-traumatic balance problems are often the result of damage to the vestibular system. Nystagmus is common in these patients, and can provide insight into the damage that has resulted from the trauma.

Objective. To present a systematic overview of published literature regarding nystagmus in patients with TBI.

Methods. Nine databases and platforms were searched during October 2016 for articles published between 1996 and 2016. Studies of any research design and published in English that focused on nystagmus in patients with TBI were considered for inclusion. A total of 110 articles were screened once duplicates had been removed, and 29 full-text articles were assessed. Eleven articles were included in the quality appraisal phase (using the McMaster tool), after which 10 articles were included in this review.

Results. This review describes nystagmus in 713 patients, and all articles reviewed described the type of assessment method that was used. However, the results lacked comprehensive data regarding the assessment, measurement and description of nystagmus in TBI patients, or the possible link and relationship between nystagmus and TBI.

Conclusions. This systematic review indicated that: $(i)$ there is a growing body of evidence that benign paroxysmal positional vertigo should be considered during the medical examination of all patients suffering from head trauma; (ii) all patients with TBI should undergo visual (eye movement) and vestibular examination; and (iii) future studies should include quantitative measurements of eye movements and nystagmus.
\end{abstract}

S Afr Med J 2017;107(11):957-964. DOI:10.7196/SAMJ.2017.v107i11.12472

Traumatic brain injury (TBI) is a non-degenerative, non-congenital injury that occurs when a sudden trauma causes structural and/or physiological damage to the brain. ${ }^{[1]} \mathrm{TBI}$ is a health and socioeconomic concern worldwide owing to its impact not only on the individual person suffering from the injury, but also on the national health and economic growth of a country. ${ }^{[2]}$ The declared global prevalence rate of TBI of 200 per 100000 people per year is considered to be an underestimate, ${ }^{[3]}$ probably because mild TBI, also referred to as concussion, is often undiagnosed, misdiagnosed and/or untreated. ${ }^{[4,5]}$ The prevalence of TBI is estimated to be lower in high-income countries than in low- and middle-income countries (LMICs)..$^{[2]}$

TBI is especially prominent in LMICs facing a high preponderance of risk factors for causes of TBI, and with inadequately prepared health systems to address the associated health outcomes. In the Western hemisphere, TBI is the leading cause of mortality in persons $<45$ years of age. ${ }^{[6]}$ Population-based studies in LMICs such as South Africa (SA), Taiwan and India suggest even higher rates. This is mainly because of road traffic accidents. Indeed, males in South-East Asia and Africa have the highest and second-highest incidences, respectively, of fatalities related to road traffic injuries in the world, and it may be assumed that a significant proportion, if not the majority, of these deaths can be attributed to TBI. ${ }^{[6]}$ According to the National Institute of Occupational Health of SA, ${ }^{[7]}$ the incidence of TBI in SA is about 316 per 100000 population per year. The reported number of new cases of TBI in SA is about 89000 per year, making SA one of the countries with the highest rates of new cases in the world. ${ }^{[8]}$

Irrespective of the incidence and prevalence of TBI, it is a complex injury with a broad spectrum of symptoms and disabilities, including communication and cognitive impairment. ${ }^{[9]}$ The effect of TBI on a person and his or her family can be devastating.
TBI results in a cascade of physical, emotional, neurological and sleep-related symptoms that include headaches, tinnitus, light-headedness, dizziness, blurred vision, fatigue, irritability and memory disorders. ${ }^{[10-12]}$ Between $30 \%$ and $65 \%$ of people with TBI suffer from some form of dizziness and/or have balance problems at some point during their recovery. ${ }^{[1,13]}$

Balance is the ability to maintain one's centre of mass over its base of support. ${ }^{[14]}$ With a well-functioning balance system (vestibular system), a person can see clearly while moving, identify orientation with respect to gravity, determine direction and speed of movement, and make automatic postural adjustments to maintain posture and stability in various conditions and activities. ${ }^{[14,15]}$

The area most associated with balance is the region of the inner ear known as the vestibular system. The vestibular system consists of a complex sensory organisation that involves communication between the central and peripheral vestibular systems as well as the ocular system, the postural muscles, the brainstem and the cerebellum to regulate and control balance and sense of motion. ${ }^{[16,17]}$

In patients with TBI, post-traumatic dizziness or balance problems are often the result of damage to the vestibular system. ${ }^{[14,15]}$ The severity of post-traumatic balance problems depends on the location, type and severity of the brain injury. A dysfunctioning vestibular system results in symptoms such as dizziness, vertigo (an illusionary sensation of spinning of oneself or the environment), nausea, difficulty focusing on specific objects (which affects the ability to read or watch television, drive a car and read road signs), a sense of falling over while walking, and difficulty walking on uneven surfaces or in the dark. ${ }^{[12,18]}$ Symptoms resulting from vestibular disorders can diminish quality of life and affect all aspects of a person's activities of daily living such as walking, driving, going shopping, meeting 
with friends and working. They can also contribute to mental health disorders such as anxiety and depression and have a negative effect on a person's work and ability to concentrate. ${ }^{[19]}$

Disorders of the vestibular system can result from damage to either the peripheral or the central vestibular system. The peripheral vestibular system consists of the vestibular apparatus, which is divided into five end-organs. These are the three semicircular canals (superior, posterior and horizontal) that sense rotational head movement or angular acceleration in the yaw, pitch and roll planes, and the two otolith organs (saccule and utricle) that sense linear acceleration, head tilt and gravity. ${ }^{[1]}$ These end-organs provide peripheral sensory input and in conjunction with the central systems, provide the brain with feedback regarding the movements of the body and head. Effective co-ordination of activities and balance involves a complex integration of the sensory, motor-programming and musculoskeletal systems. ${ }^{[20]}$ Dysfunction along any pathway in the vestibular system can lead to symptoms such as dizziness and/ or vertigo. ${ }^{[18]}$

The central vestibular system has four functional connections from the vestibular nuclei, namely:

- Cerebellar connections that regulate body balance and posture and connect to the ocular motor neurons ${ }^{[16]}$

- Connections with higher centres for conscious perception of changes in posture and movements, and the mediation of appropriate adjustments of voluntary actions ${ }^{[16]}$

- Connections to the back muscles that contribute to the control of posture and adjust reflexes of the body in response to changes in position and movement ${ }^{[16]}$

- Connections to the ocular muscles that mediate the important vestibulo-ocular reflex through which control is exerted over involuntary eye movements that are triggered by movements of the head. ${ }^{[16]}$

The main types of vestibular injuries associated with TBI are benign paroxysmal positional vertigo (BPPV) (the most common peripheral vestibular disorder), labyrinthine injury and traumatic endolymphatic hydrops. Other injuries include brainstem and cerebellum injuries. ${ }^{[21]}$ One of the most important considerations when assessing vestibular disorders is the presence and description of nystagmus. ${ }^{[21]}$

Nystagmus is defined as involuntary, repetitive to-and-fro eye movements initiated by a drift of the eye away from the object of interest or focus. ${ }^{[22]}$ Nystagmus occurs when the part of the brain or inner ear that regulates eye movement and eye positioning does not function correctly, resulting in the involuntary, rapid movement of the eyes. In TBI, the shearing forces in the brain due to the injury or trauma can often cause damage to the vestibular system, leading to nystagmus. ${ }^{[1]}$

Nystagmus can be described based on its presence or absence, the position of the eye while the nystagmus is observed, the intensity (rate) of the nystagmus, the direction of the nystagmus (based on the slow-phase component) and whether it is present with or without a fixation point for the person to focus on. Nystagmus can be either spontaneous (occurring without any movement) or induced by external factors such as head and body movements.

There are two categories of nystagmus, namely congenital and acquired. Acquired nystagmus can be caused by a disease (e.g. vestibular neuritis), head injury (e.g. TBI) or a neurological pathology (e.g. acoustic neuroma). There are several different types of pathological, acquired nystagmus, of which the most common are spontaneous nystagmus (with or without fixation), positional or positioning nystagmus (occurring with or after head or body movement), gaze-evoked nystagmus (only present in specific eccentric eye gaze positions), optokinetic nystagmus (only abnormal with lowered velocity of eye movements), pendular nystagmus (independent eyeball motion with vertical, horizontal and torsional nystagmus), seesaw nystagmus (alternating elevation and intorsion of one eye and simultaneous depression and extorsion of the other), and periodic alternating nystagmus, which is a strictly horizontal nystagmus that predictably oscillates in direction, amplitude and frequency.

The direction of the nystagmus is defined by the direction of its quick phase. Nystagmus can occur in any direction (horizontal, vertical or torsional) or in a combination of these directions, and it has several different possible waveforms. ${ }^{[13]}$ The type and direction of the nystagmus are determined by the pathophysiology or cause of the pathology. Detailed assessment of the nystagmus is therefore a critical component in the diagnosis of patients with vestibular disorders and the key to successful management of these patients in an attempt to improve their quality of life.

The effects of nystagmus on a person differ depending on the type and intensity of the nystagmus. Individuals with acquired nystagmus would generally have difficulty reading or scrolling on a computer, watching television and reading fine print; they would have poor depth perception, decreased balance, and difficulty walking on uneven surfaces or crossing a busy street, and might have difficulty maintaining eye contact. People with nystagmus, and with balance problems in general, may be more affected by emotional and physical factors such as stress, tiredness, nervousness or unfamiliar surroundings than people without these problems. These factors have a negative impact on the person's mental health and vocation.

Not only does SA have a high incidence of TBI, ${ }^{[8]}$ it also has the highest rate of new cases reported worldwide. ${ }^{[6]}$ This, together with TBI's diverse effect on the individual, underscores the importance of obtaining a clear description of the nystagmus that could be present to improve the diagnosis and treatment of patients with any degree of TBI. There is currently a paucity of literature describing the presence and specific type of nystagmus in patients with TBI, as well as the possible link between the severity of TBI and the intensity of the nystagmus.

\section{Objective}

The primary purpose of this research was to use a systematic review with a multiple perspective to describe and analyse the literature that examined articles describing nystagmus in patients with TBI from 1996 to 2016. The multiple perspective used included the following:

- A description of the participants (demographic information)

- A description of the assessment method/s used to measure nystagmus (high or low technology)

- A description of the nystagmus observed in these patients (eye position, type, intensity, direction and fixation)

- The optimal time to measure nystagmus in patients with TBI

- The possible link between the severity of the TBI and the intensity of the nystagmus.

The year in which the article was published, as well as the journal in which it was published, was also noted.

\section{Methods}

\section{Search strategy}

This systematic search aimed to obtain a comprehensive list of published articles (including grey literature) on nystagmus in patients with TBI.

The search strategy followed the population, exposure and outcome (PEO) format and employed keywords and Medical Subject Heading 
(MeSH) terms related to each of these three components. The keywords used in the systematic review are depicted in Table 1. Search concept combinations were 'TBI AND nystagmus, 'TBI AND nystagmus AND assessment', 'TBI AND nystagmus AND assessment AND vestibular', and 'TBI AND nystagmus AND assessment AND vision'.

Nine databases and platforms (WorldCat, Medline, PaperFirst, ScienceDirect, SA ePublications and Journal Collection, BioOne, JSTOR Health and General Sciences, Collection, and JSTOR Life Sciences Collection) were considered relevant and searched during October 2016 for articles published between 1996 and 2016 using the keywords and MeSH terms shown in Table 1. Google Scholar was used to search the reference lists of the included articles for additional relevant articles that might meet the inclusion criteria (grey literature).

\section{Study eligibility}

All relevant published studies that used either qualitative or quantitative study designs were eligible for inclusion. Review articles, editorials and opinion pieces that did not contain primary data were excluded. For inclusion, studies also needed to have been published in English between January 1996 and October 2016 and be directly related to nystagmus and patients with TBI. This time period is significant because the US Congress passed the Traumatic Brain Injury Act in 1996 (P.L. 10-166) and the bill was signed by President Clinton on 29 July 1996. US Federal funds were subsequently awarded to states by means of a competitive grant process with the aim of developing, expanding and improving access to service delivery for individuals with TBI and their families. The result was an increased amount of research, and subsequently publications, on
TBI. Only studies that comply with the criteria as set by the PEO components were considered for inclusion in this review. A summary of the eligibility criteria is presented in Table 2 .

\section{Population}

The current review covered all studies that included participants with TBI. No exclusions were made based on participant criteria in terms of the type, severity or duration of the TBI.

\section{Exposure}

The review included articles with information on the assessment of nystagmus in TBI patients. All assessment methods (both highand low-technology types of assessments) were included and are important for describing the outcomes of the review.

\section{Outcomes}

To meet the main aim of the review, namely to investigate nystagmus in patients with TBI, four sub-aims were identified:

1. Describing nystagmus in patients with TBI in terms of:

- position: primary or gaze-related nystagmus

- type: pendular or jerk nystagmus

- intensity: rapid or slow

- direction: horizontal, vertical or rotatory nystagmus

- with or without fixation: whether the nystagmus is present with or without fixation

2. Describing current assessment methods used to measure nystagmus in patients with TBI

3. Establishing how soon after the onset of TBI nystagmus should be measured

Table 1. Search terms and keywords used based on each of the PEO components

\begin{tabular}{|c|c|c|c|}
\hline PEO component & Search concept & Search keywords (including MeSH terms) & Truncation symbols used \\
\hline Population & TBI & $\begin{array}{l}\text { TBI; traumatic brain injury; concussion; brain } \\
\text { trauma; head trauma; brain injury; head injury; } \\
\text { closed head injury; closed brain injury; closed } \\
\text { head trauma; closed brain trauma }\end{array}$ & $\begin{array}{l}\text { traumatic brain injur*; brain } \operatorname{traum}^{*} \text {; head } \\
\text { traum}^{*} \text {; brain injur }{ }^{*} \text {; head injur }{ }^{*} \text { closed head } \\
\text { injur*; closed brain injur*; closed head traum*; } \\
\text { closed brain traum* }\end{array}$ \\
\hline Exposure & Assessment & $\begin{array}{l}\text { assessment; test; evaluation; Frenzel; } \\
\text { postuography; VNG; ENG; videonystagmography; } \\
\text { eletronystagmography }\end{array}$ & assess $^{*} ;$ test $^{*} ;$ evaluat $^{*} ;{ }^{\star}$ nystag $^{*}$ \\
\hline \multirow[t]{3}{*}{ Outcome } & Nystagmus & nystagmus & \\
\hline & Vestibular & vestibular; dizziness; vertigo & $\operatorname{dizz}^{*}$ \\
\hline & Vision & $\begin{array}{l}\text { visual acuity; gaze stabilization; GST; dynamic } \\
\text { visual acuity; DVA }\end{array}$ & \\
\hline
\end{tabular}

Table 2. Inclusion and exclusion criteria based on each of the PEO components

\begin{tabular}{|c|c|c|c|}
\hline PEO component & Inclusion criteria & Exclusion criteria & Rationale \\
\hline Population & $\begin{array}{l}\text { Patients with TBI, regardless of time } \\
\text { of onset of nystagmus, gender, age } \\
\text { or ethnicity }\end{array}$ & $\begin{array}{l}\text { Patients without TBI, including } \\
\text { patients with nystagmus but without } \\
\text { TBI }\end{array}$ & $\begin{array}{l}\text { Focus of the review is on nystagmus } \\
\text { in patients with TBI }\end{array}$ \\
\hline Exposure & $\begin{array}{l}\text { All studies were included that had } \\
\text { a primary focus on assessment or } \\
\text { testing (high or low technology) }\end{array}$ & $\begin{array}{l}\text { Studies dealing with intervention or } \\
\text { rehabilitation }\end{array}$ & $\begin{array}{l}\text { To describe the outcomes, the } \\
\text { assessment of nystagmus in TBI } \\
\text { patients needs to be determined }\end{array}$ \\
\hline Outcome & $\begin{array}{l}\text { Studies included must describe at } \\
\text { least one of the four outcomes of } \\
\text { the review }\end{array}$ & $\begin{array}{l}\text { Studies not describing at least one of } \\
\text { the review's outcomes }\end{array}$ & $\begin{array}{l}\text { This review aims to describe } \\
\text { nystagmus in patients with TBI; } \\
\text { studies that do not contribute to at } \\
\text { least one of the review's aims will } \\
\text { not be included in the review }\end{array}$ \\
\hline
\end{tabular}


4. Describing the relationship between the severity of the TBI and the severity of the nystagmus.

Only studies that described at least one of these outcomes were included in the review.

\section{Data extraction and synthesis}

A four-phase process was used to assess studies for inclusion in this review and is presented as a PRISMA flow diagram in Fig. 1.

\section{Phase 1: Identification}

Two reviewers (HdC and AN) independently screened all the identified potential studies at title level. Both reviewers are dually qualified as speech-language therapists and audiologists, and have over 10 years' clinical experience. The reviewers discussed their recommendations and were in agreement about the studies to be excluded at this level of the selection process.

\section{Phase 2: Screening}

The two reviewers ( $\mathrm{HdC}$ and $\mathrm{AN}$ ) used the same procedure as in phase 1 to independently screen studies at abstract level to determine the relevance of each study for inclusion in this review. Consensus was reached on inclusion after the results of the screening process had been discussed. A Cronbach's alpha score of 0.99 was obtained, which indicated a high internal consistency. The third reviewer (JB) independently screened $20 \%$ of the articles $(n=16)$ at abstract level, and although the third reviewer was blind to the scores, $100 \%$ agreement with the first two reviewers on the scores allocated to each reviewed study was obtained.

\section{Phase 3: Eligibility and data extraction}

Full-text articles were retrieved and independently assessed by both reviewers (HdC and $\mathrm{AN}$ ) to determine their inclusion in the critical appraisal stage of the review. Data were extracted independently by both reviewers ( $\mathrm{HdC}$ and $\mathrm{AN}$ ) and subsequently recorded onto a custom-designed data extraction spreadsheet. Consensus was reached between the reviewers regarding minor discrepancies in the data extraction process. The following information was extracted verbatim and recorded onto the data extraction spreadsheet from each study that was included:

- Publication data: author, year, aim/s of the study and country of study

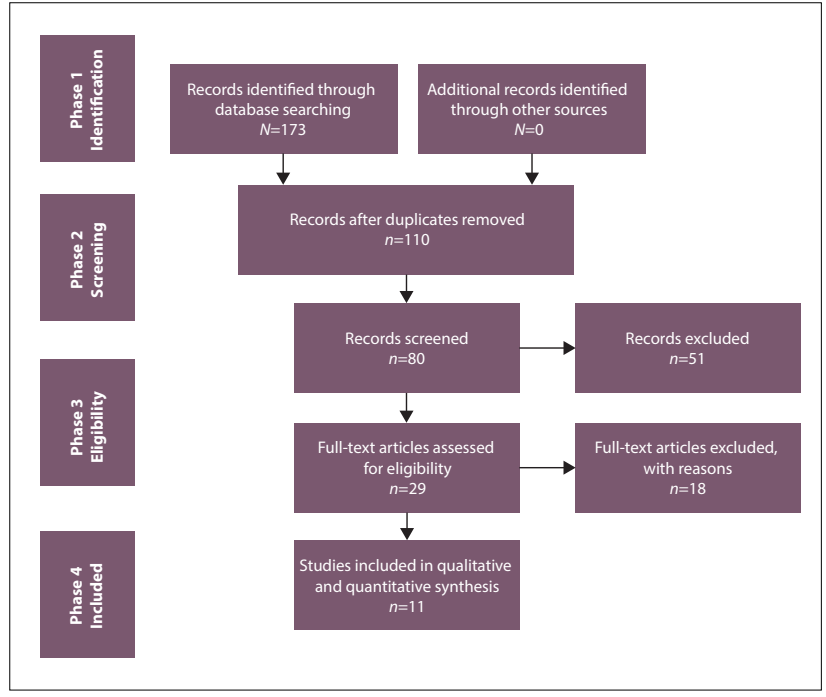

Fig. 1. PRISMA diagram depicting the study selection process.
- Participants in the study: age and gender of patients with TBI

- Intervention: details of the intervention described in the study

- Control: details of the control conditions

- Outcomes: description of nystagmus as described in terms of the four aims of the review.

Twenty-nine full-text articles were retrieved and assessed for eligibility according to the data extraction list of inclusion criteria. Of these 29 studies, 18 did not meet the inclusion criteria and were excluded for various reasons, such as study referring to TBI, but focusing more on migraine $(n=2)$, surgery $(n=2)$, genetic causes $(n=1)$, neck or head pain $(n=1)$, cognitive deficits $(n=2)$, epilepsy $(n=1)$, alcohol intoxication $(n=1)$, cerebral palsy $(n=2)$, ocular or neurological diseases $(n=2)$, acoustic trauma $(n=1)$, general brain injury $(n=1)$, syndromes $(n=1)$, and stroke and neuroimaging $(n=1)$.

The reviewers (HdC and AN) had an initial agreement score of 93\% (27 out of 29 articles) and then discussed the two articles on which they did not initially agree. Upon discussion, consensus was reached and they identified 11 studies for review (HdC and AN). All 11 studies were checked by the third reviewer (JB) and found suitable for inclusion in the review (with an agreement score of 100\%).

\section{Phase 4: Inclusion}

A total of 11 studies were included in this review, based on the fourphase assessment process used to determine the eligibility of each study.

\section{Critical appraisal}

The type of research design was not a criterion for this review, and as such, the design-generic McMaster critical appraisal tool was selected for critical appraisal. ${ }^{[23]}$ The McMaster has a total of 16 questions, of which only 14 are quantifiable. A binary scoring system was used $(1=$ yes; $0=$ no). Since question 5 was not applicable (none of the appraised studies were intervention studies), questions $5 \mathrm{a}, 5 \mathrm{~b}$ and $5 \mathrm{c}$ were not appraised and were therefore not included in the score. There remained 11 articles that could be used. Each article was subsequently appraised and allocated a score out of 11, which was then converted to a percentage.

All 11 included articles were independently reviewed and scored by reviewers $\mathrm{HdC}$ and $\mathrm{AN}$ to ensure inter-rater reliability. Inter-rater reliability was calculated with the following formula, and a reliability score of $98.34 \%$ was obtained.

$$
\begin{aligned}
& \frac{\text { Number of agreed scores }}{\text { Number of items } \times \text { Number of articles }} \times \frac{100}{1} \\
& \frac{119}{121} \times \frac{100}{1}=98.34 \%
\end{aligned}
$$

The agreed scores for each article are set out in Table 3 . The third reviewer (JB) then read three studies $(27 \%)$ that had been preselected by the first two reviewers based on their scores - the lowest score (55\%), a middle score (72\%) and one of the highest scores (91\%). Although the third reviewer was blind to the scores, $100 \%$ agreement with the first two reviewers on the scores allocated to each reviewed study was obtained.

The critical appraisal scores ranged between $5 / 11$ (55\%) to $10 / 11$ (91\%). The critical appraisal of all 11 articles revealed an average score of $79 \%$. There were only minor differences in the methodological scores between the remaining 10 articles, which resulted in a high average score. The McMaster tool assisted the reviewers (HdC, $\mathrm{AN}$ and JB) to filter out low-scoring articles and guided them to exclude the one study that scored $<60 \%$ and therefore did not make 
Table 3. Quality score of reviewed articles in chronological order (1997-2016)

\begin{tabular}{|c|c|c|c|c|c|c|c|c|c|c|c|c|c|c|}
\hline \multirow[b]{2}{*}{ Author/s } & \multirow[b]{2}{*}{ Year } & \multicolumn{11}{|c|}{ McMaster questions ${ }^{\star}$} & \multirow[b]{2}{*}{ Score } & \multirow[b]{2}{*}{ Percentage } \\
\hline & & 1 & 2 & $3 \mathbf{a}$ & $3 \mathbf{b}$ & $4 a$ & $4 b$ & $6 a$ & $6 b$ & $6 c$ & $6 d$ & 7 & & \\
\hline Hughes and Proctor ${ }^{[24]}$ & 1997 & 0 & 1 & 1 & 1 & 1 & 1 & 0 & 1 & 1 & 0 & 1 & $8 / 11$ & 72 \\
\hline Bolaños et al ${ }^{[25]}$ & 2004 & 1 & 1 & 1 & 1 & 1 & 1 & 1 & 1 & 1 & 0 & 1 & $10 / 11$ & 91 \\
\hline Gordon et al. ${ }^{[26]}$ & 2004 & 1 & 1 & 1 & 1 & 1 & 1 & 1 & 1 & 1 & 0 & 1 & $10 / 11$ & 91 \\
\hline Chelikh et al. ${ }^{[27]}$ & 2005 & 1 & 1 & 1 & 1 & 1 & 1 & 0 & 1 & 1 & 0 & 1 & $9 / 11$ & 82 \\
\hline Jackson et al. ${ }^{[28]}$ & 2007 & 1 & 1 & 1 & 1 & 1 & 1 & 1 & 1 & 1 & 0 & 1 & $10 / 11$ & 91 \\
\hline Woo et al..$^{[29]}$ & 2008 & 1 & 0 & 1 & 1 & 1 & 1 & 0 & 1 & 1 & 0 & 1 & $8 / 11$ & 72 \\
\hline Johnson $^{[30]}$ & 2009 & 0 & 1 & 1 & 1 & 1 & 1 & 0 & 1 & 1 & 0 & 1 & $8 / 11$ & 72 \\
\hline Scherer et al. ${ }^{[31]}$ & 2011 & 1 & 1 & 1 & 0 & 1 & 1 & 1 & 1 & 1 & 0 & 1 & $9 / 11$ & 82 \\
\hline Alvarez et al. ${ }^{[32]}$ & 2012 & 1 & 1 & 1 & 1 & 1 & 1 & 1 & 1 & 1 & 0 & 1 & $10 / 11$ & 91 \\
\hline $\operatorname{Liu}^{[33]}$ & 2012 & 1 & 0 & 0 & 1 & 1 & 1 & 1 & 1 & 1 & 0 & 1 & $8 / 11$ & 72 \\
\hline Yunusov et al..$^{[34]}$ & 2014 & 1 & 0 & 0 & 1 & 1 & 1 & 0 & 0 & 1 & 0 & 1 & $6 / 11$ & 55 \\
\hline Average score & & & & & & & & & & & & & $8.7 / 11$ & 79 \\
\hline
\end{tabular}

${ }^{*}$ McMaster questions: $1=$ Was the purpose stated clearly? 2 = Was the relevant background literature reviewed? $3 \mathrm{a}=$ Was the sample described in detail? $3 \mathrm{~b}=$ Was the sample size justified? $4 \mathrm{a}=$ Were the outcome measures reliable? $4 \mathrm{~b}=$ Were the outcome measures valid? $6 \mathrm{a}=$ Were results reported in terms of statistical significance? $6 \mathrm{~b}=$ Were the analysis method $(\mathrm{s})$ appropriate? $6 \mathrm{c}=$ Was clinical importance reported?; $6 \mathrm{~d}$ = Were drop-outs reported? 7 = Was the conclusion appropriate, given the study methods and results?

a significant contribution to the review. ${ }^{[35]}$ (Articles with a score of $\geq 60 \%$ were considered satisfactory, ${ }^{[35]}$ so the article that scored $55 \%{ }^{[34]}$ was excluded from the review. All the other studies scored between $72 \%$ and $91 \%$.)

The results of the extracted data will be discussed next.

\section{Results}

This section presents information in two broad categories, namely the demographics of the study and the PEO components. The data from the PEO components include population (number, age and gender of the patients, as well as TBI diagnosis), exposure (assessment method) and outcome (description of the nystagmus, time to measure the nystagmus and the relationship between nystagmus and TBI). Table 4 sets out a synopsis of the results.

\section{Demographics of the studies}

With regard to study demographics, all but one (conducted in 1997) of the 10 studies included were conducted after 2000, with six studies taking place between 2004 and 2009 and three between 2011 and 2012. This can probably be attributed to the development of technologically advanced tools and assessment methods in recent years. ${ }^{[36]}$ As mentioned above, after the passing of the TBI Act in 1996, US federal funds were awarded for developing, expanding and improving access to service delivery for individuals with TBI and their families in the USA, which significantly increased research in this field.

Five of the reviewed studies were published in the USA, and one each in Mexico, Israel, France, Korea and China. Of the 10 studies, only two were conducted in LMICs (Mexico and China). This highlights the need for more data from this context, given the high frequency of TBI.

There were no obvious trends in the 10 reviewed studies in terms of similar authors or publishing journals, with only two journals (Otology \& Neurotology and The Laryngoscope) containing two or more published studies on the topic.

\section{PEO components}

The findings of the reviewed studies in terms of PEO components (population, exposure and outcome) will now be discussed in more detail and presented in chronological order $(1997$ - 2012). The results of the systematic review clearly indicate the lack of comprehensive information about the description of nystagmus in the 713 patients with TBI included. All 10 of the reviewed studies discussed nystagmus to some degree, although the condition may not have been the main focus of the study. Only a limited number of research studies reported information on nystagmus in patients with TBI, as only 10 studies could be identified for this review.

\section{Participants}

As determined by the inclusion criteria, all 713 patients had a diagnosis of TBI. They had a wide range of ages (19-91 years), with a mean age of 45.6 years; 386 were male and 243 female, with gender not being reported for the remaining 84 . No clear difference between the gender groups was reported.

\section{Exposure}

'Exposure' in this review refers to the assessment method that was used in evaluating nystagmus in patients with TBI. The assessment method can either be high technology or low technology. High technology refers to computer-based equipment such as a highvelocity/infrared camera or other device to measure and record a patient's eye movement, specifically nystagmus. ${ }^{[37]}$ High-technology equipment can detect and measure nystagmus as small as one degree per second, which cannot be seen with the naked eye. ${ }^{[38]}$

Low technology includes all bedside assessments to document a patient's eye movement, such as Frenzel glasses and even questionnaires. ${ }^{[39]}$ Low-technology measures are typically more reliant than high-technology ones on the examiner's skill and experience in detecting nystagmus. When the nystagmus is too small for the examiner to see with the naked eye, it can easily be missed. ${ }^{[38]}$

In this review, six out of the ten studies relied on low-technology measures to report nystagmus, while only four used high-technology equipment. Although the exact type of equipment was not mentioned, it can be assumed that the results from the high-technology measures would be more accurate than the low-technology (Frenzel glasses or naked eye) assessment of the presence of nystagmus. ${ }^{[40]}$ Of the four studies that utilised high-technology equipment to measure the nystagmus, three were conducted in the USA. Neither of the studies in the LMICs made use of high-technology measures.

\section{Outcome}

The outcomes of the review involved reporting specifically on: $(i)$ the description of nystagmus found in patients with TBI; (ii) the time to 

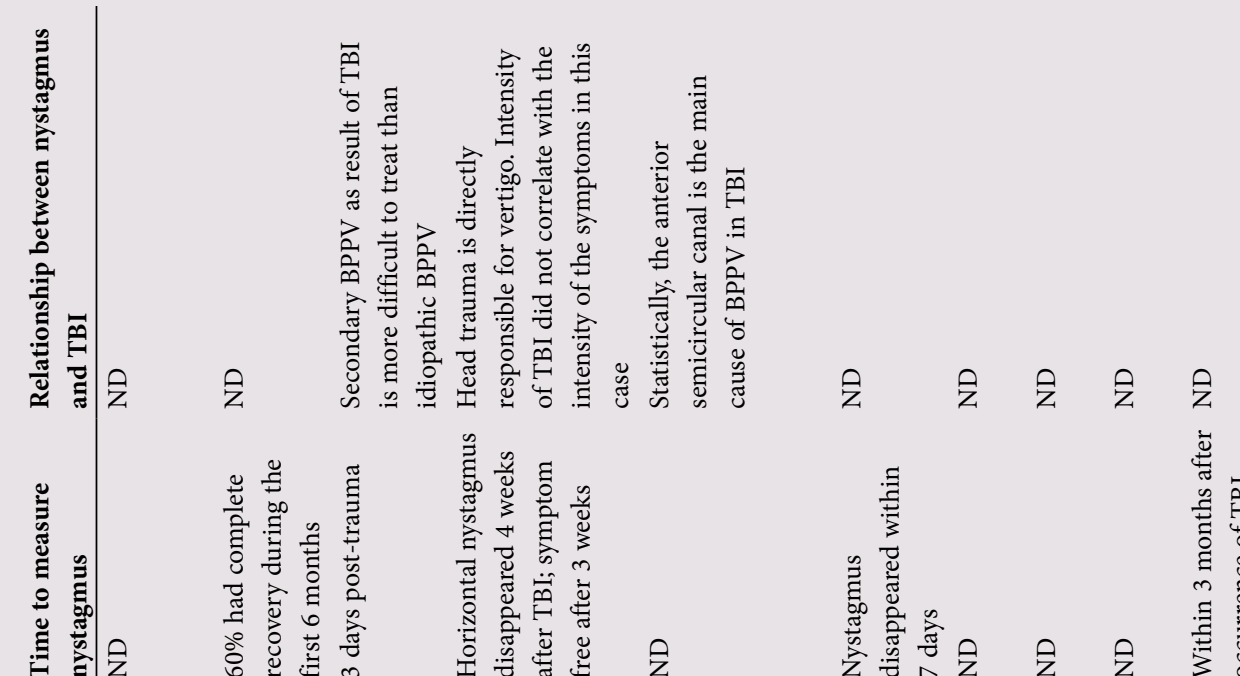

合合合合

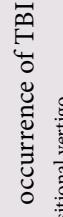

安

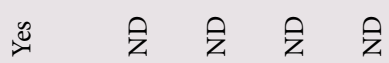

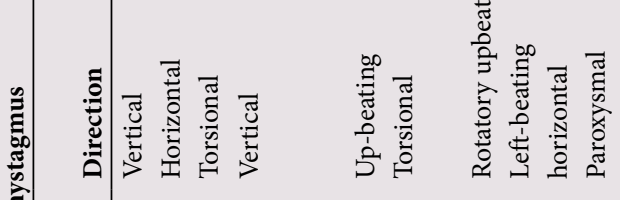

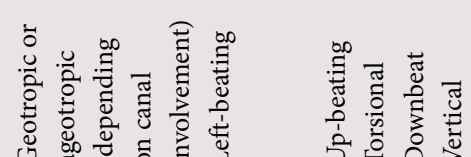

窇

咅是

合文

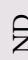

合令令

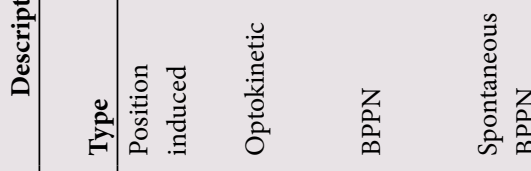

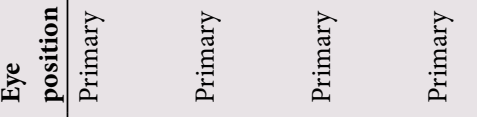

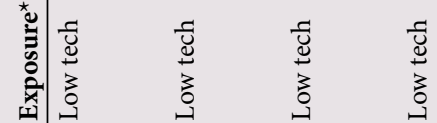

ङัँ

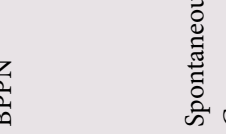

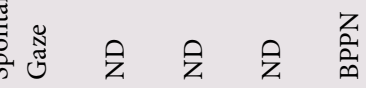

害

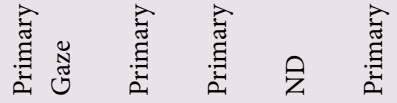

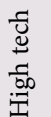

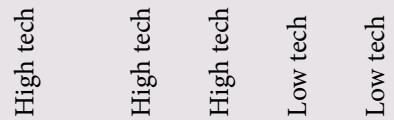

ㅇํำ

$\stackrel{\star x}{0}$

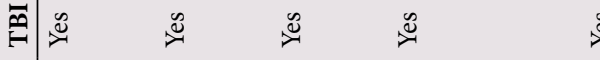

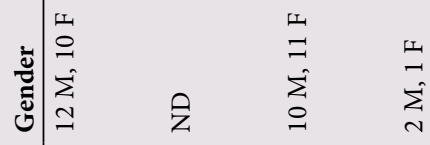

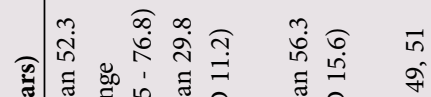

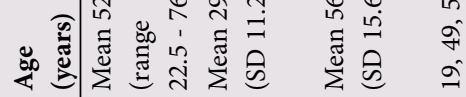

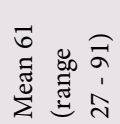

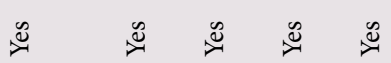

$z$ 4

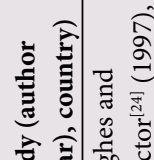

原

$\frac{2}{2} \frac{5}{8}$

$\sqrt{x}$

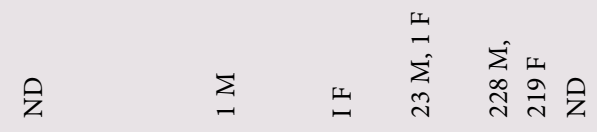

品 in 亲

m

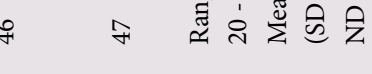


measure nystagmus after the TBI incident; and (iii) the relationship between the severity of the TBI and the degree of nystagmus.

\section{Description of the nystagmus}

The nystagmus found in patients with TBI was categorised according to eye position, type of nystagmus, intensity of the nystagmus, direction of the nystagmus and whether it was measured with or without fixation.

A primary (centre) eye position was reported in nine out of the ten studies, with one study reporting both primary and gaze-related eye positions. Only one study did not report on the patient's eye position during testing. The position of the eye while measuring nystagmus is important, as it can indicate different pathologies based on when the nystagmus was evoked.

The type of nystagmus in these reviews varied greatly and no single type could be identified. Two studies reported two separate types of nystagmus observed in the participants. The results included benign paroxysmal positional nystagmus $(n=4)$, spontaneous nystagmus $(n=42)$, position-induced nystagmus $(n=41)$, optokinetic nystagmus $(n=41)$ and gaze-evoked nystagmus $(n=41)$. The remaining three studies did not report on the type of nystagmus observed or evoked during assessment.

The intensity of nystagmus is measured in degrees per second. ${ }^{[40]}$ This is only possible when high-technology equipment is used. ${ }^{[38]}$ Although four of the studies reported using high-technology equipment, none reported on the intensity or degree of nystagmus found in the patients with TBI. Only three studies reported on the presence of fixation during testing, with only one study using fixation and the remaining two testing without the presence of a fixation point.

\section{Time to measure the nystagmus}

The best time to measure nystagmus after the TBI incident was explored in this section. It is important to know the best time to measure the nystagmus, as the symptoms can vary and disappear altogether over time. ${ }^{[15]}$ Of the 10 studies reviewed, five did not discuss the best time to measure nystagmus after TBI. The studies that did discuss it reported varying data, ranging from 3 days after trauma to a complete recovery in most patients within 6 months.

\section{Relationship between nystagmus and TBI}

In this section the focus was on trying to establish whether there was any relationship between the intensity of the nystagmus and the severity of the TBI. Seven of the ten studies did not discuss this relationship, while of the remaining three studies, one indicated that traumatic BPPV is more difficult to treat than idiopathic BPPV. The other two studies provided important data about this relationship: one ${ }^{[27]}$ indicated that head trauma was directly responsible for vertigo and that the intensity of TBI did not correlate with the intensity of symptoms/nystagmus in this case, while the other ${ }^{[28]}$ mentioned that, statistically speaking, the anterior semicircular canal is the main cause of BPPV in TBI.

The results of the review indicated a lack of comprehensive data regarding the assessment and measurement of nystagmus in TBI patients, as well as on the possible link and relationship between nystagmus and TBI.

\section{Discussion}

In this systematic review, 10 articles were reviewed to compile information regarding nystagmus in patients with TBI. The review indicated that most components related to nystagmus - especially its description and measurement in patients with TBI - are not well documented.
The type of equipment used for measuring and assessing nystagmus is important for the outcome of the review, as only high-technology equipment can measure its intensity. ${ }^{[38]}$ In some cases, where the intensity of the nystagmus is very low, low-technology assessment methods can also not determine its direction. ${ }^{[39]}$ The fact that lowtechnology measures were used in most of the reviewed studies could explain the lack of data on the intensity of the nystagmus.

Determining the direction of nystagmus can assist in differentially diagnosing a patient. It is important to note the direction (or change in direction) of the nystagmus, as this can be indicative of specific abnormalities. If the patient has a low-intensity nystagmus, lowtechnology equipment would not be able to detect or assess the direction of the nystagmus, which could lead to inaccurate data. In the 10 reviewed studies, no single direction of nystagmus was observed and the reported directions of the nystagmus included vertical, horizontal and rotatory (torsional) nystagmus, as well as up-beating, down-beating, left-beating and right-beating nystagmus. When measuring nystagmus, it is important to know whether it is present with or without a fixation point. This will assist in determining whether the lesion is central or peripheral in nature. Most peripheral lesions can result in nystagmus that can be suppressed with fixation, whereas central lesions usually result in nystagmus that is present even with fixation.

Should nystagmus not be measured early in the evaluation, it could be missed and remain undocumented. ${ }^{[41]}$ No clear indication of when nystagmus should be measured was obtained from this review.

An important recommendation noted in this review (from Scherer et al..$^{[31]}$ ) was that although some patients may appear to be asymptomatic, $30 \%$ of these asymptomatic patients had abnormal vestibular findings and nystagmus. Vestibular testing confirms a higher incidence of vestibular and oculomotor dysfunction in patients with blast-related TBI than in asymptomatic patients.

The review also highlighted the lack of data describing the measurement and assessment of nystagmus in patients with TBI and indicated the need for high-technology measures to be used when assessing these patients. Use of high-technology equipment could aid in the documentation of nystagmus in patients with TBI, which would lead to more accurate diagnosis and management. ${ }^{[37,38]}$

\section{Limitations of this review}

The findings that emerged from this review are limited to the primary purpose of this research, which was to use a systematic review with a multiple perspective to describe and analyse the literature that examined articles describing nystagmus in patients with TBI from 1996 to 2016, and generalisations therefore cannot be made outside this context. The overall approach to this review was strengthened by the development of an a priori protocol and adherence to the PRISMA statement. ${ }^{[42]}$

Other shortcomings of the review are that only studies in English and only peer-reviewed studies that were available online were considered for inclusion - clinical case reports, lecture or conference notes, books, theses or other unpublished literature were therefore not considered. Furthermore, the review question and subsequent search terms did not specifically include rehabilitation or intervention studies.

\section{Recommendations and conclusion}

This systematic review indicated that, with regard to patients with chronic recurrent vertigo after TBI:

- There is a growing body of evidence that BPPV should be considered during the medical examination of all patients suffering from head trauma. ${ }^{[30]}$ 
- All patients with TBI should undergo visual (eye movement) and vestibular examination.

- Future studies should include quantitative measurements of eye movements and nystagmus. ${ }^{[32]}$

Furthermore, it is recommended that a paediatric sample should be included in the participant group, and that studies should be conducted with high-technology assessment methods to report on the intensity and direction of the nystagmus observed in patients with TBI.

In conclusion, there is a lack of evidence regarding the description of nystagmus in patients with TBI, and more research is needed specifically on using high-technology equipment to report on the eye position, type, intensity, direction and fixation of nystagmus. Information is also needed on the optimal time for measuring nystagmus in patients with TBI and on the possible relationship between the intensity of the nystagmus and the severity of the TBI. This information would enable clinicians to obtain a better understanding of the importance of correct measurement of nystagmus in TBI patients, which could improve their care and diagnosis, as well as their quality of life.

\section{Acknowledgements. None.}

Author contributions. All three authors were equally involved in the production of the article and read the final manuscript.

Funding. None.

Conflicts of interest. None

1. Akin FW, Murnane OD. Head injury and blast exposure: Vestibular consequences. Otolaryngol Clin North Am 2011;44(2):323-334. https://doi.org/10.1016/j.otc.2011.01.005

2. Lucas S, Hoffman JM, Bell KR, Dikmen S. A prospective study of prevalence and characterization of headache following mild traumatic brain injury. Cephalalgia 2014;34(2):93-102. https://doi. org $/ 10.1177 / 0333102413499645$

3. Bryan-Hancock C, Harrison J. The global burden of traumatic brain injury: Preliminary results from the Global Burden of Disease Project. Inj Prev 2010;16:17. https://doi.org/10.1136/ip.2010.029215.61

4. Jury MA, Flynn MC. Auditory and vestibular sequelae to traumatic brain injury: A pilot study. N Z Med 4. Jury MA, Flynn MC. Auditory and vestibular sequelae to traumatic brain injury: A pilot study. $\mathrm{N}$ Z Med
J 2001;114(1134):286-288. https://www.ncbinlm.nih.gov/pubmed/11480511 (accessed 10 October 2017).

5. Ruff RL, Riechers RG, Wang XF, Piero, T, Ruff SS. A case-control study examining whether neurological deficits and PTSD in combat veterans are related to episodes of mild TBI. BMJ Open 2012;2(2):1-12 deficits and PTSD in combat veterans are rel/2d
https://doi.org/10.1136/bmjopen-2011-000312

https://doi.org/10.1136/bmjopen-2011-000312
6. Naidoo D. Traumatic brain injury: The South African landscape. S Afr Med J 2013;103(9):613-614. 6. Naidoo D. Traumatic brain injury:
https://doi.org/10.7196/SAMJ.7325

7. National Institute of Occupational Health, South Africa. Traumatic brain injury (head injuries) - World Head Injury Awareness. National Health Laboratory Service: Topical Issues. http://www.noioih.ac.za/?pa ge=topical\&id=13\&rid=214 (accessed 23 November 2016).

8. Pretorius C, Broodryk M. Misconceptions about traumatic brain injuries among South African university students. S Afr J Psychiatry 2013;19(3):75-79. https://doi.org/10.7196/SAJP.436

9. Arciniegas DB, Held K, Wagner P. Cognitive impairment following traumatic brain injury. Curr Treat Options Neurol 2002;4(1):43-57. https:///doi.org/10.1007/s1 1940-002-0004-6

10. Alsalaheen BA, Mucha A, Morris LO, et al. Vestibular rehabilitation for dizziness and balance disorders alter concussion. J Neurol Phys Ther 2010;34(2):87-93. https:///doi.org/10.1097/NPT.0b013e3181dde568
after

11. Hoffer ME, Gottshall KR, Moore R, Balough BJ, Wester D. Characterizing and treating dizziness after mild head trauma. Otol Neurotol 2004;25(2):135-138. https://doi.org/10.1097/00129492-200403000-00009

12. McLeod TCV, Hale TD. Vestibular and balance issues following sport-related concussion. Brain In 2015;29(2):175-184. https://doi.org/10.3109/02699052.2014.965206

13. Lueck CJ. Nystagmus. Pract Neurol 2005;5(5):288-291. http://pn.bmj.com/content/5/5/288 (accessed 10 October 2017).
14. Shumway-Cook A, Woollacott MH. Motor Control: Theory and Practical Applications. Philadelphia: Lippincott, Williams \& Wilkins, 2001:75-91. https://doi.org/10.1097/01253086-199620010-00023

15. Hanes DA, McCollum G. Cognitive-vestibular interactions: A review of patient difficulties and possible mechanisms. J Vestib Res 2006;16(3):25-91. https://www.ncbi.nlm.nih.gov/pubmed/17312336 (accessed
mestibed 10 October 2017).

16. Cuschieri A. Anatomy of the peripheral and central vestibular system. Capsula Eburnea 2009;4(16):11-24. https://doi.org/10.3269/1970-5492.2009.4.16

17. Khan S, Chang R. Anatomy of the vestibular system: A review. NeuroRehabilitation 2013;32(3):437-443. https://doi.org/10.3233/NRE-130866

18. Brennan M. Visual-vestibular interaction and treatment of dizziness: A case report. J Behav Optom 2012;23(3):68-72. https://www.oepf.org/journal/pdf/jbo-volume-23-issue-3-visual-vestibular-interactionand-treatment-dizziness-case-report (accessed 10 October 2017).

19. Hain TC, American Hearing Research Foundation. Post-traumatic vertigo. http://american-hearing.org/ disorders/post-traumatic-vertigo/ (accessed 25 November 2016).

20. Basford JR, Chou L, Kaufman KR, et al. An assessment of gait and balance deficits after traumatic brain injury. Arch Phys Med Rehabil 2003;84(3):343-349. https://doi.org/10.1053/apmr.2003.50034

21. Labuguen RH. Initial evaluation of vertigo. Am Fam Physician 2006;73(2):244-251. https//wwwnchi nlm.nih.gov/pubmed/16445269 (accessed 10 October 2017).

22. Stahl JS, Leigh RJ. Nystagmus. Curr Neurol Neurosci Rep 2001;1(5):471-477. https://doi.org/10.1007/ s11910-001-0109-4

23. Law M, Stewart D, Pollock N, Letts L, Bosch J, Westmorland M, McMaster University. Critical Review Form - Qualitative Studies (Version 2.0) Ontario: McMaster University, 2007. https://www.canchild.ca/ system/tenon/assets/attachments/000/000/359/original/qualform.pdf (accessed 10 October 2017).

24. Hughes CA, Proctor L. Benign paroxysmal positional vertigo. Laryngoscope 1997;107(5):607-613. https://doi.org/10.1097/00005537-199705000-00010

25. Bolaños I, Lozano D, Cantú C. Internuclear ophthalmoplegia: Causes and long-term follow-up in 65 patients. Acta Neurol Scand 2004;110(3):161-165. https://doi.org/10.1111/j.1600-0404.2004.00278.x

26. Gordon CR, Levite R, Joffe V, Gadoth N. Is posttraumatic benign paroxysmal positional vertigo different from the idiopathic form? Arch Neurol 2004;61(10):1590-1593. https://doi.org/10.1001/ archneur.61.10.1590

27. Chelikh L, Timoshenko AP, Bertholon P, Tringali S, Martin C. Combined horizontal and posterior canal benign paroxysmal positional vertigo in three patients with head trauma. Ann Otol Rhinol Laryngol 2005;114(2):105-110. https://doi.org/10.1177/000348940511400204

28. Jackson LE, Morgan B, Fletcher JC, Krueger WWO. Anterior canal benign paroxysmal positional vertigo: An underappreciated entity. Otol Neurotol 2007;28(2):218-222. https://doi.org/10.1097/01. mao.0000247825.90774.6b

29. Woo H, Song S, Kim Y, Bai CH. Pneumolabyrinth without temporal bone fracture: Different outcomes for hearing recovery. Laryngoscope 2008;118(8):1464-1466. https://doi.org/10.1097/ MLG.0b013e318172ab03

30. Johnson EG. Clinical management of a patient with chronic recurrent vertigo following a mild traumatic brain injury. Case Rep Med 2009 (2009), Article ID 910596. https://doi.org/10.1155/2009/910596

31. Scherer MR, Burrows H, Pinto R, et al. Evidence of central and peripheral vestibular pathology in blast-related traumatic brain injury. Otol Neurotol 2011;32(4):571-580. https://doi.org/10.1097/ MAO.0b013e318210b8fa

32. Alvarez TL, Kim EH, Vicci VR, Dhar SK, Biswal BB, Barrett AM. Concurrent vision dysfunctions in convergence insufficiency with traumatic brain injury. Optom Vis Sci 2012;89(12):1740-1751. https://doi, org/10.1097/OPX.0b013e3182772dce

33. Liu H. Presentation and outcome of post-traumatic benign paroxysmal positional vertigo. Acta Otolaryngol 2012;132(8):803-806. https://doi.org/10.3109/00016489.2012.657359

34. Yunusov F, Park J, Huh YE, Kim H, Kim J. Mystery case: Pendular see-saw nystagmus as a delayed complication of traumatic brain injury. Am Acad Neurol 2014;82(4):147-148. https://doi.org/10.1212/ WNL 0000000000000358

35. Audet N, Gagnon R, Ladouceur R, Marcil M. How effective is the teaching of critical analysis of scientific publications? Review of studies and their methodological quality. CMAJ 1993;148(6):945-952. https:// www.ncbi.nlm.nih.gov/pubmed/8448709 (accessed 10 October 2017).

36. Ferreiro G, Carda S, Moslavac S, Rabini A. Technological advances in instrument assessment and rehabilitation. Biomed Res Int 2015 (2015), Article ID 264067. https://doi.org/10.1155/2015/264067

37. Manso A, Gananca MM, Caovilla HH. Vestibular rehabilitation with visual stimuli in peripheral vestibular disorders. Braz J Otorhinolaryngol 2016;82(2):232-241. https://doi.org/10.1016/.bjorl.2015.05.019

38. Yao J, Tai Z, Yin Z. A new measure of nystagmus acuity. Int J Ophthalmol 2014;7(1):95-99. https://doi. org/10.3980/j.issn.2222-3959.2014.01.17

39. Blohm A. A survey of audiologists' clinical practices and their formal education and training in the assessment and management of adults with vestibular pathologies. AuD thesis, Department of Audiology, Speech-Language Pathology and Deaf Studies, Towson University, Md., 2013.

40. Miles RD, Zapala DA. Vestibular function measurement devices. Semin Hear 2015;36(1):49-74. https:// doi.org/10.1055/s-0034-1396926

41. Jacobson GP, Shephard NT. Balance Function Assessment and Management. 2nd ed. San Diego, Calif.: Plural Publishing, 2014

42. Moher D, Liberati A, Tetzlaff J, Altman DG, the PRISMA Group. Preferred reporting items for systematic reviews and meta-analyses: The PRISMA statement. Ann Intern Med 2009;141(4):264-269. https://doi. org/10.1371/journal.pmed.1000097

Accepted 8 June 2017 